\title{
Cardiac arrest after intracoronary injection of diltiazem
}

\author{
J. Allal, D. Coisne and R. Barraine \\ Service de Cardiologie A, Centre Hospitalier Régional et Universitaire de Poitiers, \\ Cité Hospitalière de la Milétrie, Poitiers, France
}

The calcium inhibitor diltiazem (Tildiem ${ }^{\circledR}$ ) can be given by intracoronary injection as a means of treating coronary spasm [1]. Recent studies have shown that it depresses both sinus function and auriculoventricular conduction, irrespective of whether it is administered orally or intravenously, and both in the present and absence of an underlying sinus disorder [2-4]. To our knowledge, no case of cardiac arrest has previously been reported, though we have also described the present case briefly in French [5].

\section{Case}

R.L., a man aged 63, was referred to our Department for dilatation of a stenosis of Segment II of the right main coronary artery. Prior to dilatation, the electrocardiograym was normal. After the first inflation of a $3 \mathrm{~mm}$ balloon using 4 atmospheres pressure, the patient experienced anginal pain with modification of the ECG in the lower field; the artery was occluded at the site of the stenosis. Two further inflations were performed without success. The artery was however recanalized following intracoronary administration of $4 \mathrm{mg}$ of isosorbid dinitrate, thus confirming the spastic nature of the occlusion. Some minutes later, however, the artery was reoccluded; diltiazem was injected into the vessel in a dose of 50 gamma $/ \mathrm{kg}$. Immediately on completion of the injection cardiac arrest occurred with a flat ECG tracing, necessitating immediate external cardiac massage followed by brief electrostimulation of the ventricle. After ten minutes of effort, a normal ECG tracing reappeared with a normal PR interval. A further injection of $2 \mathrm{mg}$ isosorbide dinitrate relieved the spasm. Two further dilatations were performed (6 atmospheres for 2 minutes) resulting in complete normalization of the condition. Six months later, the patient remains free of symptoms and a maximum exertion test with thallium scintigraphy has detected no abnormality.

\section{Discussion}

Two comments on this case may be made. Firstly, it seems clear that diltiazem given by intracoronary injection is less effective than isosorbid dinitrate for the treatment of coronary

Correspondence to: Prof. R. Barraine, Service de Cardiologie A, Centre Hospitalier Régional et Universitaire de Poitiers, Cité Hospitalière de la Milétrie, 86021 Poitiers, France.

0924-6479/90/\$03.50 @ 1990 Elsevier Science Publishers B.V. (Biomedical Division) 
spasm (see also [1]. Secondly, in view of the severity of the complication experienced by this patient, intracoronary administration of this compound should be employed only with the greatest caution; this applies particular when one is dealing with the right coronary artery, since the passage of the drug into the artery serving the sinus node was probably responsible for precipitating the cardiac arrest.

\section{References}

1 Bertrand ME, Dupuis BA, Lablanche JM et al. Coronary hemodynamics following intravenous or intracoronary injection of diltiazem in man. J Cardiovasc Pharmacol 1982;4:695-699.

2 Brent ML, Schroeder JS, Mason IW. Comparative clinical electrophysiologic effects of diltiazem, verapamil and nifedipine: a review. Am J Cardiol 1982;43:629-635.

3 Ishikawa $\mathrm{T}$, Inamura $\mathrm{T}$, Koiwaya $\mathrm{Y}$ et al. Atrio-ventricular dissociation and sinus arrest induced by oral diltiazem. N Engl J Med;309:1124-1125.

4 akubowski AT, Mizgala HF. Effect of diltiazem overdose. Am J Cardiol 1987;60:932-933.

5 Allal J, Coisne D, Barraine R. Arrêt cardiaque après injection intracoronarienne de diltiazem. Press Méd 1990;21:428. 